\title{
Coded Single Input Channel for Color Pattem Recognition in Joint Transform Comelator
}

\author{
Man Ho Jeong* \\ Department of Laser \& Optical Information Engineering, Cheongju University, \\ 36 Naeduk-dong, Sangdang-gu, Cheongju 360-764, Korea
}

(Received September 8, 2011 : revised October 21, 2011 : accepted October 26, 2011)

\begin{abstract}
Recently, we reported a single input channel joint transform correlator for the color pattern recognition which decomposes the input color image into three R, G, and B gray components and adds those components into a single gray image in the input plane. This technique has the merit of a single input channel instead of three input channels. However, we found this technique has some problems with discrimination impossibility in the case of a simple primary color pattern which results in the same gray level through the addition process. Thus, we propose a modified coding technique which selectively recombines the decomposed three R, G, and B gray components instead of the simple adding process. Simulated results show that the modified coding technique can accurately discriminate a variety of kinds of color images.
\end{abstract}

Keywords: Color pattern recognition, Joint transform correlator, Color difference OCIS codes : (070.0070) Fourier optics and signal processing; (070.5010) Pattern recognition

\section{INTRODUCTION}

Pattern recognition can be applied to various kinds of fields, for example facial appearance, fingerprints, handwriting, and character recognition [1-7]. These kinds of pattern recognition and target tracking applications can be achieved by the joint transform correlator (JTC) [8-16]. The introduction of color information in pattern recognition by the JTC is usually made by means of a multi-channel correlation technique that decomposes the source and the target color images into three red, green, and blue RGB channels. The correlation is made separately for each channel, and arithmetic or logical point-wise operations can be used to derive the final output [17-20]. The multi-channel JTC methods perform the correlation process in parallel for all the color channels that compose the image by the utilization of coherent optical correlators that are illuminated simultaneously with a few coherent sources, each having a different wavelength. The output plane consists of a set of superimposed correlation distributions that must be analyzed independently and composed together to render the detection decision. A disadvantage of this approach is that it requires three different channels, which increases the system cost. For example, a spatial light modulator (SLM) is needed at each channel, two lenses are required at each channel, and an extra beam splitter and a mirror are also needed. The concept of multi-channel single output color JTC configuration was initially proposed by Deutsch to overcome such disadvantages [21]. However, the multi-channel single output color JTC has to take into account the separation between the input target image and the reference image. Deutsch [21] found that the separation between the input target image and the reference image for the same color must be the same and also larger than the sum of the widths of these images in order to prevent any unwanted overlapping between correlation outputs. To yield sharp correlation peaks, Alam et al. proposed a fringe-adjusted JTC based on the Newton-Raphson algorithm [22]. However, they still used multi-channel input and single output JTC to improve correlation discrimination.

We proposed previously a technique of color pattern recognition by decomposing the color image into three color components and adding those components into a single gray image in the input plane [23]. This new technique requires a single input channel instead of three input channels However, this technique has some problems with discrimination impossibility in the case of simple color patterns such as red,

\footnotetext{
*Corresponding author: manho@cju.ac.kr

Color versions of one or more of the figures in this paper are available online.
} 
green, and blue which result in the same gray level through the addition process. Thus, we propose a modified coding technique of selectively recombining the decomposed three $\mathrm{R}, \mathrm{G}$, and $\mathrm{B}$ gray images instead of a simple addition process. We present the simulated results to show that our newly proposed technique can accurately recognize and discriminate color difference. In the Section II, conventional multi-channel color pattern recognition JTC and its basic theory is presented. In Section III, the proposed new method of encoded single input channel and single output color pattern recognition JTC system are described in detail. Section IV describes simulation results for the color pattern recognition, and finally, some comments are contained in the conclusion.

\section{SINGLE-CHANNEL SINGLE OUTPUT JTC}

We present a new single-channel single output JTC for color pattern recognition of the color image. This method can achieve color pattern recognition by decomposing the color image into three color components (red, green, and blue) and recombining those components into a single gray image in the input plane instead of using these three color components. This new technique needs a single input channel instead of three input channels and single output CCD camera, thus a simple JTC can be used.

Now, Fig. 1 shows the transformation of the three decomposed color components of the color images into a single gray image. In this process, first, three color components (red, green, and blue) are transformed into the corresponding gray components, and finally these three gray components are added together to form a single gray image. Equation (1) expresses these recombined single gray images for the reference and target, respectively.

$$
r_{R G B}=I_{R}+I_{G}+I_{B}, t_{r g b}=I_{r}+I_{g}+I_{b}
$$

where each component of Eq. (1) represents the gray image of the corresponding red, green, or blue component. Figure 2 shows the optical structure of the basic JTC system for the single-channel and single output color pattern recognition. Thus,

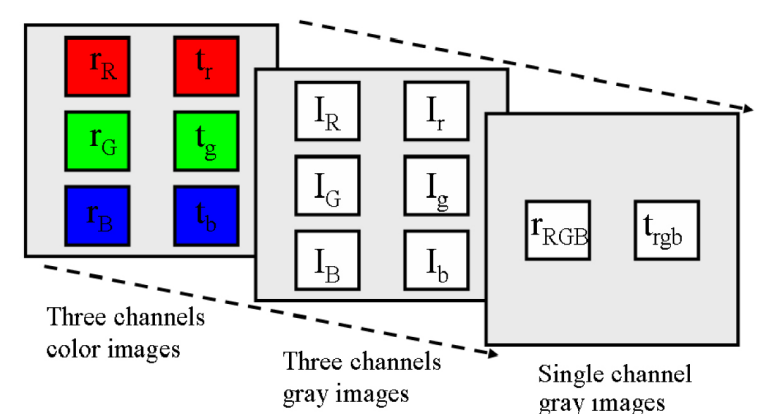

FIG. 1. Transform of the three decomposed color components of the color images into a single gray image. the input joint images $u(x, y)$ can be expressed in a simple form as

$$
u(x, y)=r_{R G B}\left(x+x_{0}, y\right)+t_{r g b}\left(x-x_{0}, y\right)
$$

After the input joint image on the LCD1 passes through the lens L1, $u(x, y)$ will be Fourier transformed as Eq. (3).

$$
\begin{aligned}
U\left(f_{x}, f_{y}\right) & =R\left(f_{x}, f_{y}\right) \exp \left[j 2 \pi\left(f_{x} x_{0}\right)\right] \\
& +T\left(f_{x}, f_{y}\right) \exp \left[-j 2 \pi\left(f_{x} x_{0}\right)\right]
\end{aligned}
$$

Therefore, JPS, the intensity of the interfered light is captured by a light detector on the Fourier plane. The JPS contains noise and DC components which degrade the correlated signal obtained on the output plane in the second stage. The DC components should be blocked to obtain a wellcorrelated signal. The blocking of the DC components may be implemented by use of a Fourier-plane image-subtraction technique. This paper also uses the non-linear JTC which introduces a non-linearity parameter $k$ to compensate the non-linear component problem caused by elements such as the digital camera [15]. Equation (4) expresses the JPS without the DC components and with the non-linearity parameter $k$.

$$
\begin{aligned}
\left|U\left(f_{x}, f_{y}\right)\right|^{2}= & {\left[R\left(f_{x}, f_{y}\right) S^{*}\left(f_{x}, f_{y}\right)\right]^{k} \exp \left[j 2 \pi\left(2 x_{0}\right)\right] } \\
& +\left[R^{*}\left(f_{x}, f_{y}\right) S\left(f_{x}, f_{y}\right)\right]^{k} \exp \left[j 2 \pi\left(-2 x_{0}\right)\right]
\end{aligned}
$$

The cross-correlation output can be obtained by inverse Fourier transforming the JPS through the lens L2. The cross-correlation output displayed on the output plane through the inverse Fourier transform lens L2 can be expressed as Eq. (5).

$$
o(x, y)=[|r(x, y) \| s(x, y)|]^{k}\left[\delta\left(x+2 x_{0}, y\right)+\delta\left(x-2 x_{0}, y\right)\right]
$$

There exist only two correlation terms at the output plane in comparison with those 36 correlation terms for the conventional three input-channel JTC.

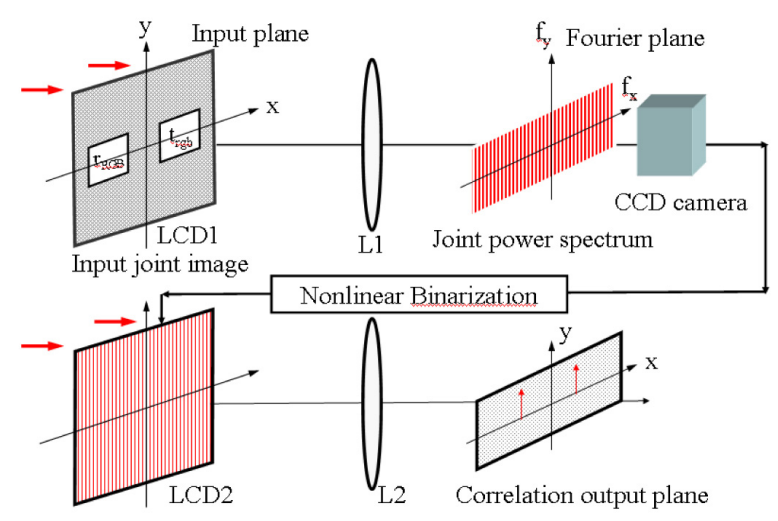

FIG. 2. Arrangement of the basic JTC for the single-channel and single output color pattern recognition. 


\section{MODIFIED CODING FOR SINGLE CHANNEL INPUT}

We previously presented useful results of color pattern recognition by using our new single input channel JTC. However, there exists some measurement limitation of our new technique. First, let's look at the case of primary single color image such as red, green, and blue instead of the mixed color image. Red color has three components $(\mathrm{R}=255$, $\mathrm{G}=0, \mathrm{~B}=0$ ) value. Green and blue image has three components $(\mathrm{R}=0, \mathrm{G}=255, \mathrm{~B}=0)$ and $(\mathrm{R}=0, \mathrm{G}=0, \mathrm{~B}=255)$, respectively. These color images have different color components, but they produce the same recombined gray scale value through the addition process expressed as Eq. (1). Thus, these primary single color images can not be discriminated by the JTC based on our previously proposed method. Now, we propose a new modified technique of coding the recombined single gray image to overcome such problem as shown on Fig. 3. Figure 3 represents two kinds of processes. First, part (a) shows our previously proposed method of obtaining the recombined single gray image through the addition process. Second, part (b) shows the modified method of obtaining a single gray image through the modified coding process. Figure 4 represents the method of obtaining a single gray input by using the modified coding technique. This modified coding technique requires two steps. First, three R, G, and B arrays of the gray images are obtained by decomposing the original color input images. Three R, G, and B arrays of the reference can be expressed as Eq. (6).

$$
I_{R}=\left(\begin{array}{ccc} 
& & \\
a_{11} & \cdots & a_{1 n} \\
\vdots & \ddots & \vdots \\
a_{m 1} & \cdots & a_{m n} \\
& &
\end{array}\right), I_{G}=\left(\begin{array}{ccc} 
& & \\
b_{11} & \cdots & b_{1 n} \\
\vdots & \ddots & \vdots \\
b_{m 1} & \cdots & b_{m n} \\
& &
\end{array}\right), I_{B}=\left(\begin{array}{ccc} 
& & \\
c_{11} & \cdots & c_{1 n} \\
\vdots & \ddots & \vdots \\
c_{m 1} & \cdots & c_{m n} \\
& &
\end{array}\right)
$$

where $a_{\mathrm{nm}}, b_{\mathrm{nm}}$, and $c_{\mathrm{nm}}$ are the elements of $\mathrm{R}, \mathrm{G}$, and B 256 $\times 256$ gray images. Second, each row of these R, G, and $\mathrm{B}$ array is selected one by one and coded in order to form a newly generated $256 \times 256$ image as shown on Fig. 4 .

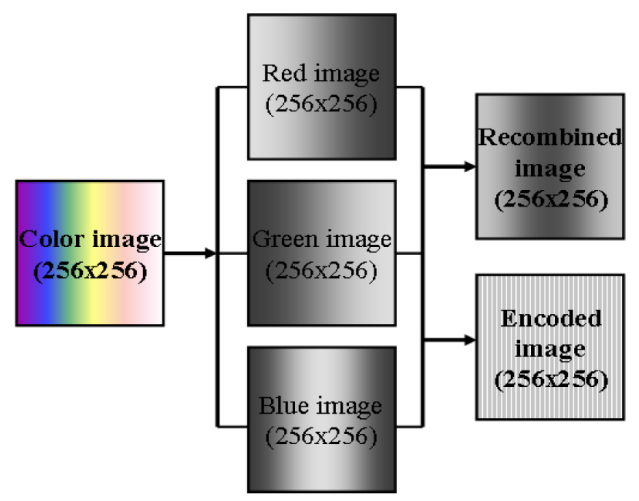

FIG. 3. Two methods of obtaining the single gray input channel image ; (a) addition process (b) coding process.

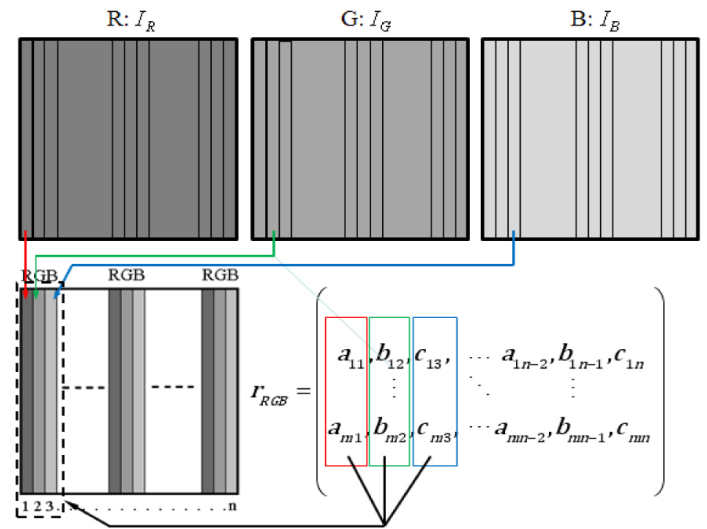

FIG. 4. Coding of R, G, and B components.

Thus, newly coded reference matrix $r_{R G B}$ has first column elements, for example as $\left[a_{11}, b_{12}, c_{13}, \cdots, a_{1 n-2}, b_{1 n-2}, c_{1 n}\right]$. Newly coded $256 \times 256$ gray image for the reference and target can be expressed as Eq. (7).

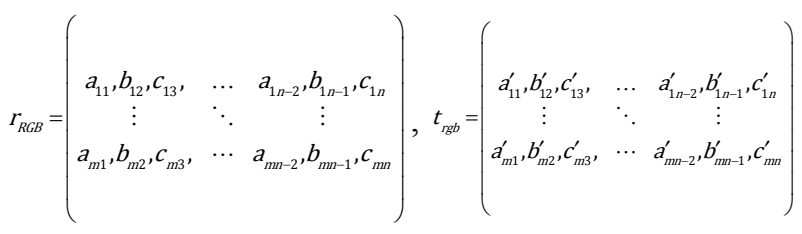

\section{SIMULATED RESULTS}

First, in Fig. 5 we prepared $256 \times 256$ mixed color images of the fruit to evaluate the performance of the proposed encoded single input channel and single output JTC. The fruits on Fig. 5 are composed of three non-zero value of R, $\mathrm{G}$, and $\mathrm{B}$ components. Figure 5(a) is the reference image, and sample 1 (Fig. 5(b)) and sample 2 (Fig. 5(c)) are the target images which are different from the reference images in the hue of the right-side pepper. As a fact, we prepared these fruit images to investigate the ability of discrimination when the color of some region of the target image is different from that of the reference. In this case, as mentioned above, the images are composed of three non-zero values of R, G, and B components. Figure 6 shows two kinds of single gray input images obtained from the original reference image. Figure 6(b) is the recombined image with addition process, and Fig. 6(c) is obtained through the coding process. Table 1 shows the correlation peaks according to these two kinds of image processing. Table 1 indicates that the correlation peaks decrease considerably when the red pepper of the fruit image shown on Fig. 5(a) changes into green and blue. For the addition process the correlation peak of the matched case is about $1.1487 \times 10^{7}$, but the correlation peaks of the mismatched case of green (sample 1) and blue peppers(sample 2) are about $1.0908 \times 10^{7}$ and $0.8948 \times 10^{7}$, respectively. Again, for the coding process the correlation 


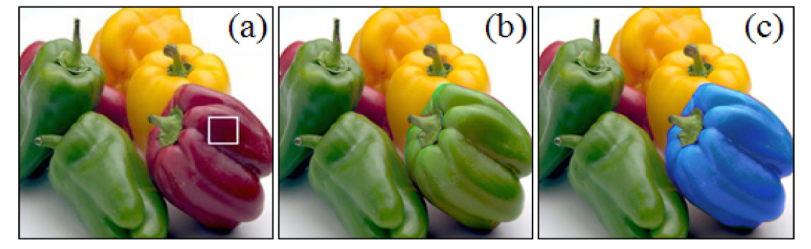

FIG. 5. 256×256 mixed color images; (a) reference (b) sample 1 (c) sample 2 .

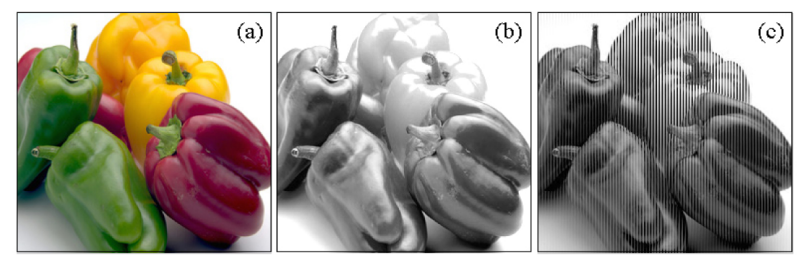

FIG. 6. Single gray images obtained from the reference image shown on Fig. 6(a) by the two different methods; (a) reference color image (b) addition process (c) coding process.

TABLE 1. Correlation peak according to the method of the single input channel

\begin{tabular}{c|c|c}
\hline \hline \multirow{2}{*}{ Input image } & \multicolumn{2}{|c}{ Correlation peak } \\
\cline { 2 - 3 } & Added method & Coded method \\
\hline Reference & $1.1487 \times 10^{7}$ & $1.2477 \times 10^{7}$ \\
\hline Sample 1 & $1.0908 \times 10^{7}$ & $1.1215 \times 10^{7}$ \\
\hline Sample 2 & $0.8948 \times 10^{7}$ & $1.0596 \times 10^{7}$ \\
\hline
\end{tabular}

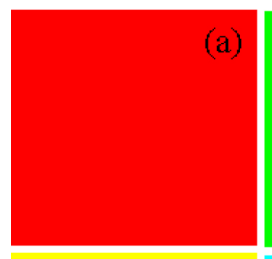

(d)

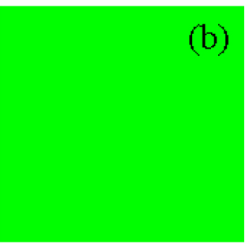

(e)

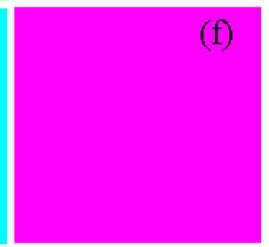

FIG. 7. 256 $\times 256$ simple color images ; (a) reference, red (b) green (c) blue (d) yellow (e) cyan (f) magenta.

peak of the matched case is about $1.2477 \times 10^{7}$, but the correlation peaks of the mismatched case of green (sample 1) and blue pepper (sample 2) are about $1.1215 \times 10^{7}$ and $1.0596 \times 10^{7}$, respectively. Therefore, we can conclude that these two methods are good for this kind of mixed color recognition.

Second, in Fig. 7 we prepared 256×256 simple color images to evaluate the performance of the proposed encoded single input channel and single output JTC. Figure 7(a) is the reference image (Red), and the others from (b), Green to (f), Magenta are the target images. In this case, these

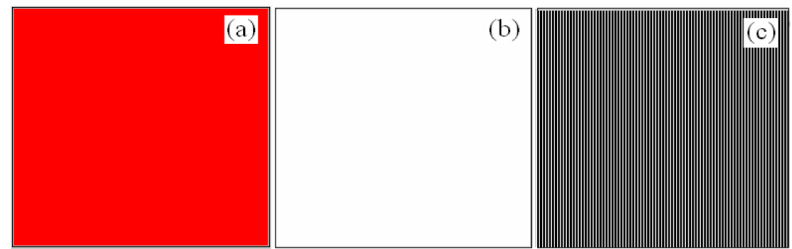

FIG. 8. Single gray images obtained from the reference image shown on Fig. 7(a) by the two different methods; (a) reference color image (b) addition process (c) coding process.

TABLE 2. Correlation peak according to the method of single input channel

\begin{tabular}{c|c|c}
\hline \hline \multirow{2}{*}{ Input image } & \multicolumn{2}{|c}{ Correlation peak } \\
\cline { 2 - 3 } & Added method & Coded method \\
\hline Ref.(Red) & $1.8494 \times 10^{6}$ & $1.8904 \times 10^{6}$ \\
\hline Green & $1.8494 \times 10^{6}$ & $1.5131 \times 10^{6}$ \\
\hline Blue & $1.8494 \times 10^{6}$ & $1.5073 \times 10^{6}$ \\
\hline Yellow & $1.8494 \times 10^{6}$ & $1.5871 \times 10^{6}$ \\
\hline Cyan & $1.8494 \times 10^{6}$ & $0.7903 \times 10^{6}$ \\
\hline Magenta & $1.8494 \times 10^{6}$ & $1.5890 \times 10^{6}$ \\
\hline
\end{tabular}

images have at least one zero value of $\mathrm{R}, \mathrm{G}$, and $\mathrm{B}$ components. For example, reference image (Red) has three components $(\mathrm{R}=255, \mathrm{G}=0, \mathrm{~B}=0)$ value. Also green and blue images have three components $(\mathrm{R}=0, \mathrm{G}=255, \mathrm{~B}=0)$ and $(\mathrm{R}=0, \mathrm{G}=0, \mathrm{~B}=255)$, respectively. These color images have different color components, but they produce the same recombined gray scale image through the addition process. Likewise, yellow, cyan, and magenta color image have different color components, but they produce the same recombined gray scale image through the addition process. Thus, we propose a new modified technique of coding the recombined single gray image to overcome such a problem. As mentioned above, this coding technique rearranges three gray images of red, green, and blue components into a single array in $\mathrm{R}, \mathrm{G}, \mathrm{B}$ order instead of using the addition process. This technique separates R, G, and B gray components for each different position in order to make a new matrix unlike that of the addition process. Figure 8 shows two kinds of single gray input images obtained from the reference image (Red). Figure 8 (b) is the recombined image with addition process, and Fig. 8(c) is obtained through the coding process. Table 2 shows the correlation peaks according to these two kinds of image processing for the simple color images. Table 2 indicates that the correlation peaks show the same value when the addition process is used, thus these simple color images can not be discriminated by the JTC based on the previously proposed addition method. On the other hand, Table 2 shows that the correlation peaks of the target color images are lower than the reference, thus we can conclude that simple color images are well discriminated by the JTC based on our newly modified coding method. Figure 9 shows the 2-D correlation peaks from the fruit images shown on Fig. 5 by using the coding process. 


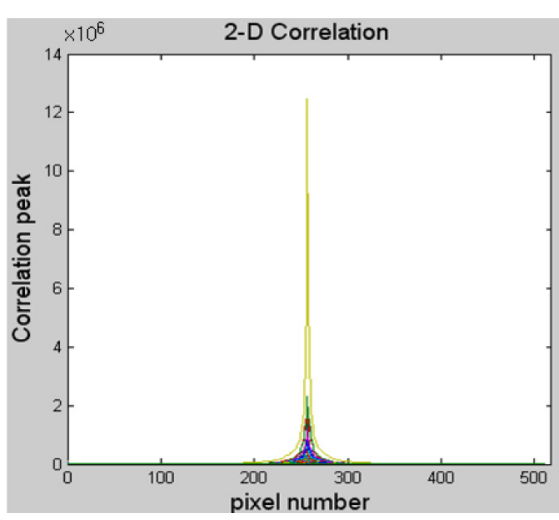

(a)

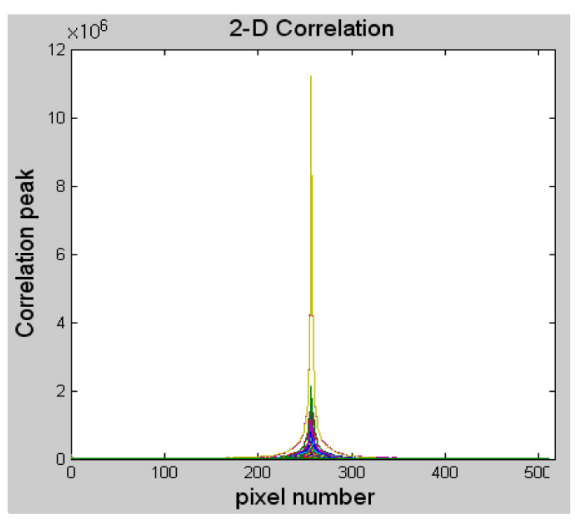

(b)

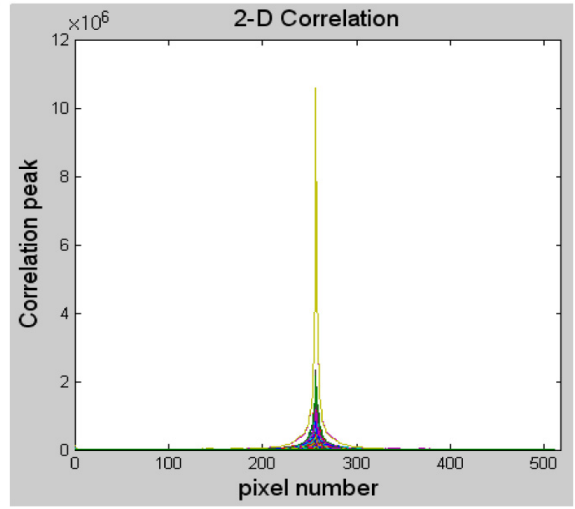

(c)

FIG. 9. 2-D diagram of the correlation peaks obtained from the fruit images shown on Fig. 6 ; (a) reference (b) sample 1 (c) sample 2.

\section{CONCLUSION}

In conclusion, the proposed modified coding technique represents an excellent single input channel JTC to overcome the problem of the complicated three input channel JTC. Moreover, the main advantage of our newly proposed JTC adopting the coding method is that it can discriminate well not only the mixed color images but also the simple color images. In detail, the final results shows that the modified coding technique solves the problems of the discrimination impossibility arising for the case of a simple primary color pattern which results in the same gray level through the addition process. Thus, we expect that our coding technique of the single input JTC color pattern can be used not only for the finding of a small section of the target such as the crowd images but also for the discrimination of a slight color difference in color measurement.

\section{REFERENCES}

1. P. D. Gianino and J. L. Horner, "Phase-only matched filtering," Appl. Opt. 23, 812-816 (1984).

2. G. G. Mu, X. M. Wang, and Z. Q. Wang, "Amplitudecompensated matched filtering," Appl. Opt. 27, 3461-3463 (1988).

3. C. J. Weaver and J. W. Goodman, "A technique for optically convolving two functions," Appl. Opt. 5, 1248-1249 (1966).

4. J. W. Goodman, Introduction to Fourier Optics, 2nd ed. (McGraw-Hill, New York, USA, 1996).

5. H. C. Lee and R. E. Gaensslen, Advances in Finger-print Technology (Elsevier, New York, USA, 1991).

6. A. Jain, L. Hong, and R. Bolle, "On-line fingerprint verification," IEEE Trans. Pattern Analysis and Machine Intell. 19, 302-314 (1997).

7. M. H. Jeong, "Analysis of fingerprint recognition characteristics based on new CGH direct comparison method and nonlinear joint transform correlator,” J. Opt. Soc. Korea 13, 445-450 (2009).

8. M. S. Alam, A. A. S. Awwal, and M. A. Karim, "Improved correlation discrimination using joint Fourier transform optical correlator," Microwave \& Opt. Technol. Lett. 4, 103-106 (1991).

9. B. Javidi, J. Li, A. H. Fazollahi, and J. Horner, "Binary nonlinear joint transform correlator performance with different thresholding methods under unknown illumination conditions," Appl. Opt. 34, 886-890 (1995).

10. M. H. Jeong, "New random and additional phase adjustment of joint transform correlator," J. Opt. Soc. Korea 14, 90-96 (2010).

11. G. Urcid-S, A. Padilla-V., A. Cornejo-R., and J. Baéz-R., "Analysis of the joint Fourier spectrum for dual input single channel rotation," Proc. SPIE 4419, 620-623 (2001).

12. X. W. Chen, M. A. Karim, and M. S. Alam, "Distortion invariant fractional power fringe adjusted joint transform correlation," Opt. Eng. 37, 138-143 (1998).

13. A. Bal, A. M. El-Sada, and M. S. Alam, "Improved fingerfrint identification with supervised filtering enhancement," Appl. Opt. 44, 647-654 (2005).

14. M. H. Jeong, "New iterative filter for fringe adjustment of joint transform correlator," J. Opt. Soc. Korea 14, 33-37 (2010).

15. B. Javidi, "Nonlinear joint power spectrum based optical correlators," Appl. Opt. 28, 2358-2367 (1989).

16. M. H. Jeong, "Binary nonlinear joint transform correlator with sinusoidal iterative filter in spectrum domain," J. Opt. Soc. Korea 14, 357-362 (2010).

17. F. T. S. Yu, Z. Yang, and K. Pan, "Polychromatic target identification with a color liquid-crystal-TV-based joint-transform correlator," Appl. Opt. 33, 2170-2172 (1994).

18. D. Mendlovic, P. Garciá-Martinez, J. Garciá, and C. Ferreira, "Color encoding for polychromatic single-channel optical pattern recognition,” Appl. Opt. 34, $7538-7544$ (1995).

19. M. Corbalan and M. S. Millan, "Color image acquisition by charge-coupled device cameras in polychromatic pattern recognition," Opt. Eng. 35, 754-758 (1996).

20. D. Mendlovic, M. Deutsch, C. Ferreira, and J. Garciá, "Single channel polychromatic pattern recognition by the use of a joint-transform correlator," Appl. Opt. 35, 6382-6389 (1996).

21. M. Deutsch, J. Garcia, and D. Mendlovic, "Multichannel singleoutput color pattern recognition by use of a joint transform correlator," Appl. Opt. 35, 6976-6982 (1996).

22. M. S. Alam, S. F. Goh, and S. Dacharaju, "Three-dimensional color pattern recognition using fringe-adjusted joint transform correlation with CIE lab coordinates," IEEE Trans. on Instru. and Measur. 59, 2176-2184 (2010).

23. M. H. Jeong, "Color pattern recognition with recombined single input channel joint transform correlator," J. Opt. Soc. Korea 15, 140-145 (2011). 